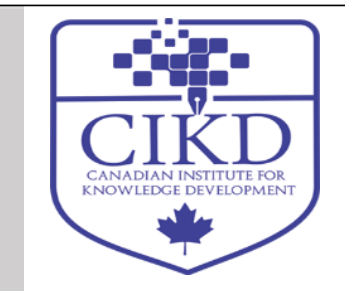

\title{
Effective Coiling of a Post-surgical Aortic Pseudoaneurysm
}

\author{
Antonio Rizza ${ }^{1 *}$, Francesco Negro² ${ }^{2}$ Cataldo Palmieri ${ }^{3}$, Sergio Berti ${ }^{4}$ \\ ${ }^{1,3,4}$ Division of Cardiology, Ospedale del Cuore, Fondazione Toscana "G. Monasterio", Massa, Italy \\ ${ }^{2}$ Division of Cardiology of Pisa, Italy
}

\begin{tabular}{ll}
\hline & ABSTRACT \\
\cline { 2 - 2 } $\begin{array}{l}\text { Keywords: } \\
\text { A 45eudoaneurysm, Coil, }\end{array}$ & $\begin{array}{l}\text { type A aortic dissection. A 3 months CT follow-up documented a significant pseudoaneurysm } \\
\text { Percutaneous intervention }\end{array}$ \\
$\begin{array}{l}\text { close to the aortic root. A percutaneous intervention was successfully performed with the } \\
\text { deployment of four Penumbra Coil inside the pseudoaneurysm, ensuring a proper exclusion } \\
\text { Received }\end{array}$ & as highlighted in long-term CT follow-up.
\end{tabular}

24 Janjuary 2021

Received in revised form

28 February 2021

Accepted

17 March 2021

*Correspondence:

rizza@ftgm.it

CCIKD Publishing

Pseudoaneurysm is usually formed when a break in the intimal layer causes blood to collect within a false lumen covered by the outer adventitia or when an injury involving the entire vessel wall causes a leak confined by surrounding tissues. They are more likely to occur due to localized intimal disease, trauma, or after surgery. However, reoperation on the thoracic aorta for aortic pseudoaneurysm is not without risk when it is attributed to hospital mortality of $6.9 \%$ [1]. Therefore, we aimed to report a 45 years old man who underwent a surgical replacement of ascending aorta and aortic arch for a type A aortic dissection 


\section{Case Presentation}

A hypertensive 45-year-old man arrived in an emergency at our first aid for an acute episode of angina associated with retrograde amnesia. Computed tomography (CT) scan revealed a Stanford type A aortic dissection extending to the left common iliac artery. The patient underwent an emergency surgical intervention with replacement of ascending aorta and aortic arch with a 4branch Vascutek Gelweave Plexus graft (Terumo, Ann Arbor, MI, USA), and debranching of the supra-aortic vessel with subsequent reimplantation of epi-aortic vessels at the tubular portion of ascending aorta. Post-operative CT scan showed a good result of the treatment with a residual dissection flap starting from the aortic arch up to the left common iliac artery.

At the 3-month follow-up, CT scan showed a $24 \times 6 \times 14 \mathrm{~mm}$ post-surgical pseudoaneurysm at the proximal anastomosis of the aortic graft, close the aortic root and non-coronary sinus, with a 10mmx5mm neck (Figure 1A, 1B, 1C).

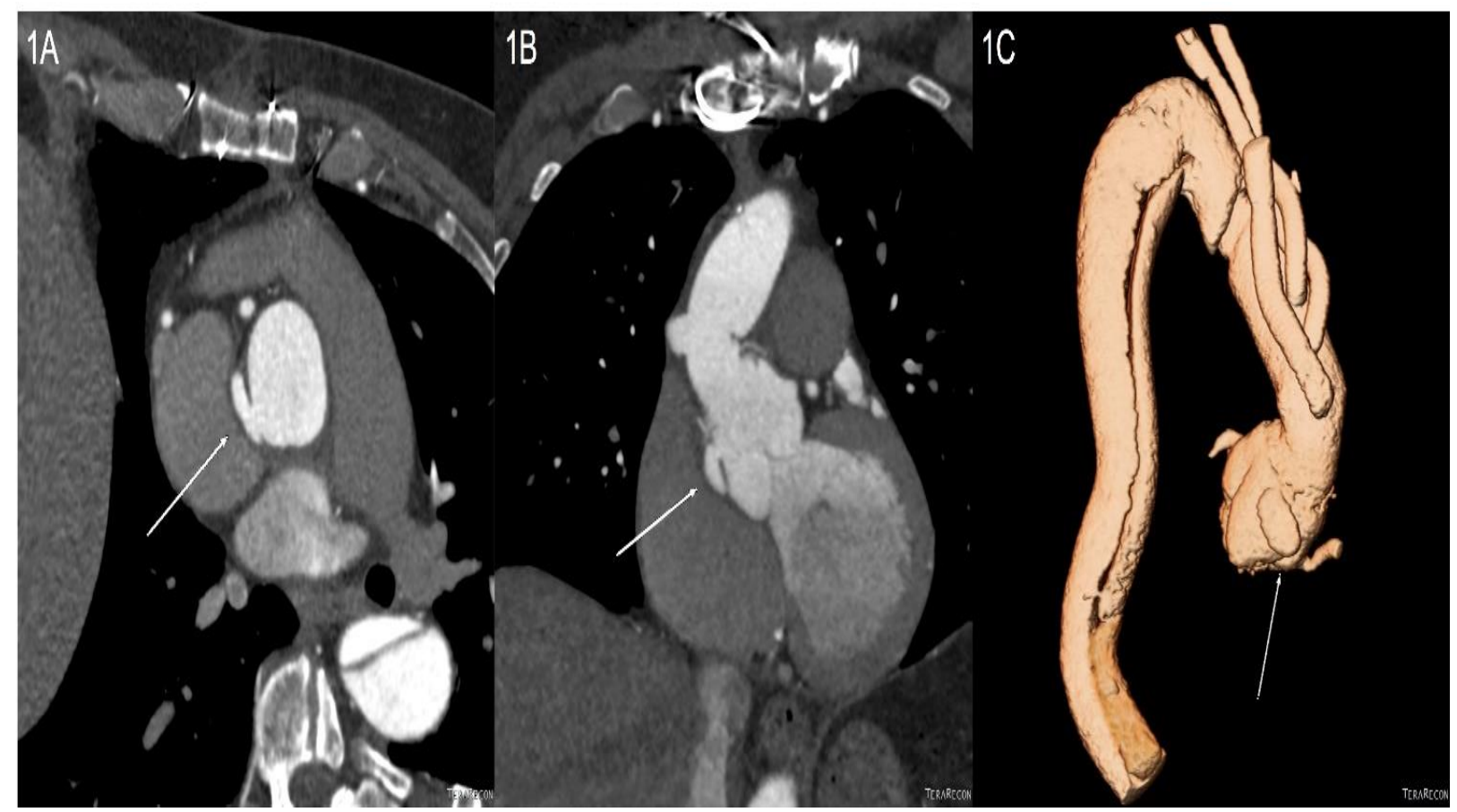

Figure 1. White arrows show the presence of the aortic pseudoaneurysm and the closeness with coronaryostia in (1A) trasversal, (1B) coronal, and (1C) 3D CT reconstructions.

Successive angiography also confirmed the non-pulsatile pseudoaneurysm in communication with an aortic root at the level of the non-coronary sinus (Figure 2A). After heart team discussion, the decision was to perform a minimally invasive percutaneous treatment. After a selective angiogram with a diagnostic catheter, the Px Slim straight microcatheter Penumbra Inc., Alameda, CA, USA) was placed in the pseudoaneurysm and four Penumbra Coil 400 $(15 \mathrm{~mm} \times 45 \mathrm{~cm}, 10 \mathrm{~mm} \times 30 \mathrm{~cm}, 7 \mathrm{~mm} \times 20 \mathrm{~cm}, 6 \mathrm{mmx} 10 \mathrm{~cm}$ - Penumbra Inc., Alameda, CA, USA) were detached inside the sac. The penumbra coils had rapidly taken shape within the targeted anatomy and packing density looks very good. The result was satisfying, and the final angiogram showed complete pseudoaneurysm exclusion, as seen in the final image (Figure 2B). 


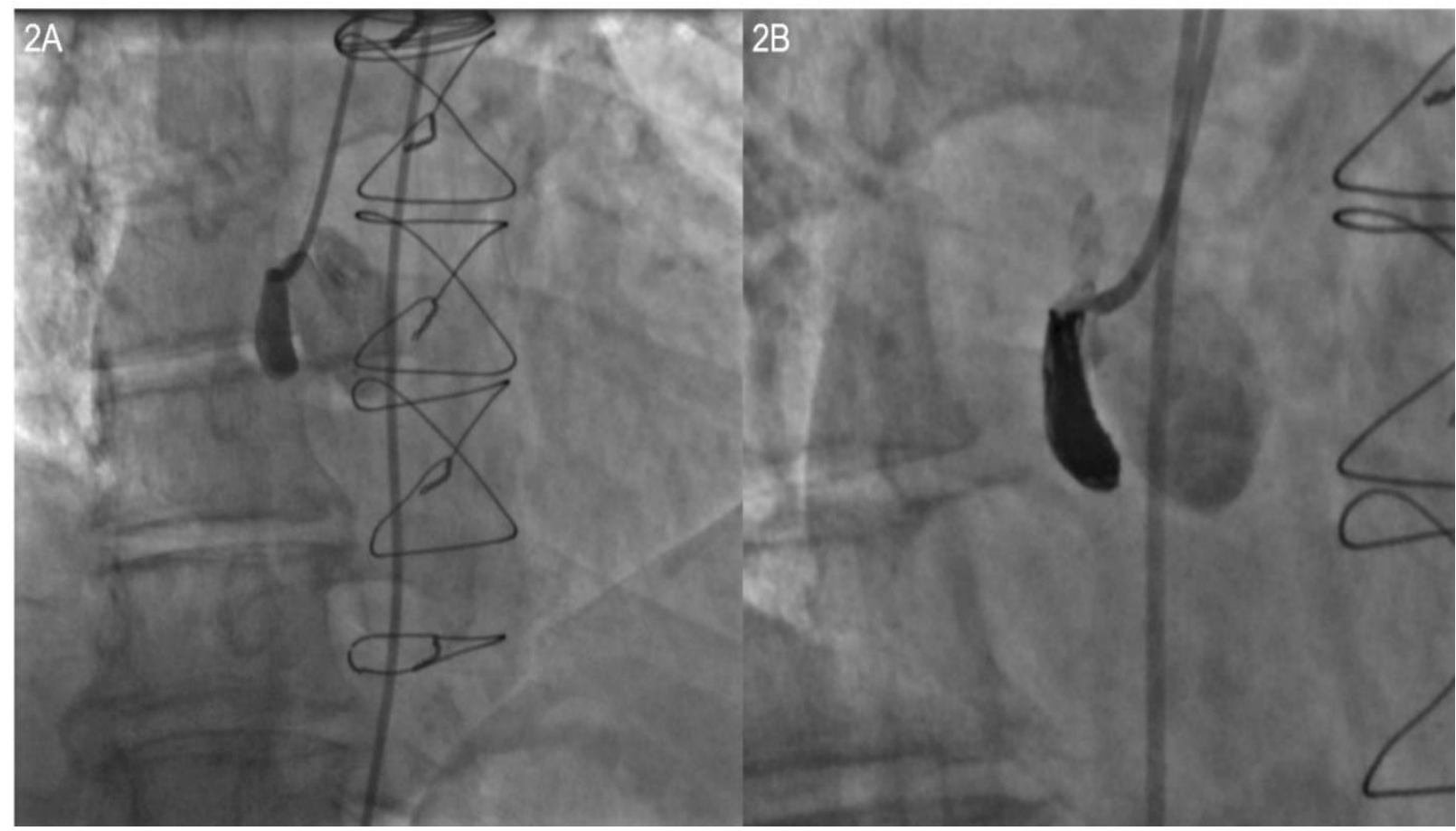

Figure 2. (2A) Basal angiography and (2B) final result after coil delivering and successful exclusion of the pseudoaneurysm.

After seven days, a thoracic endovascular aortic repair (TEVAR), with deployment of a 34x30x200cm Bolton Relay stent graft (Bolton Medical, Sunrise, FL, USA), was performed to repair the descending aorta and to exclude the residual dissection. The intervention was completed without complication. As demonstrated by the persistent exclusion of the pseudoaneurysm at 1-2-3 years CT follow-up (Figure 3A, 3B, 3C), technical success was achieved.

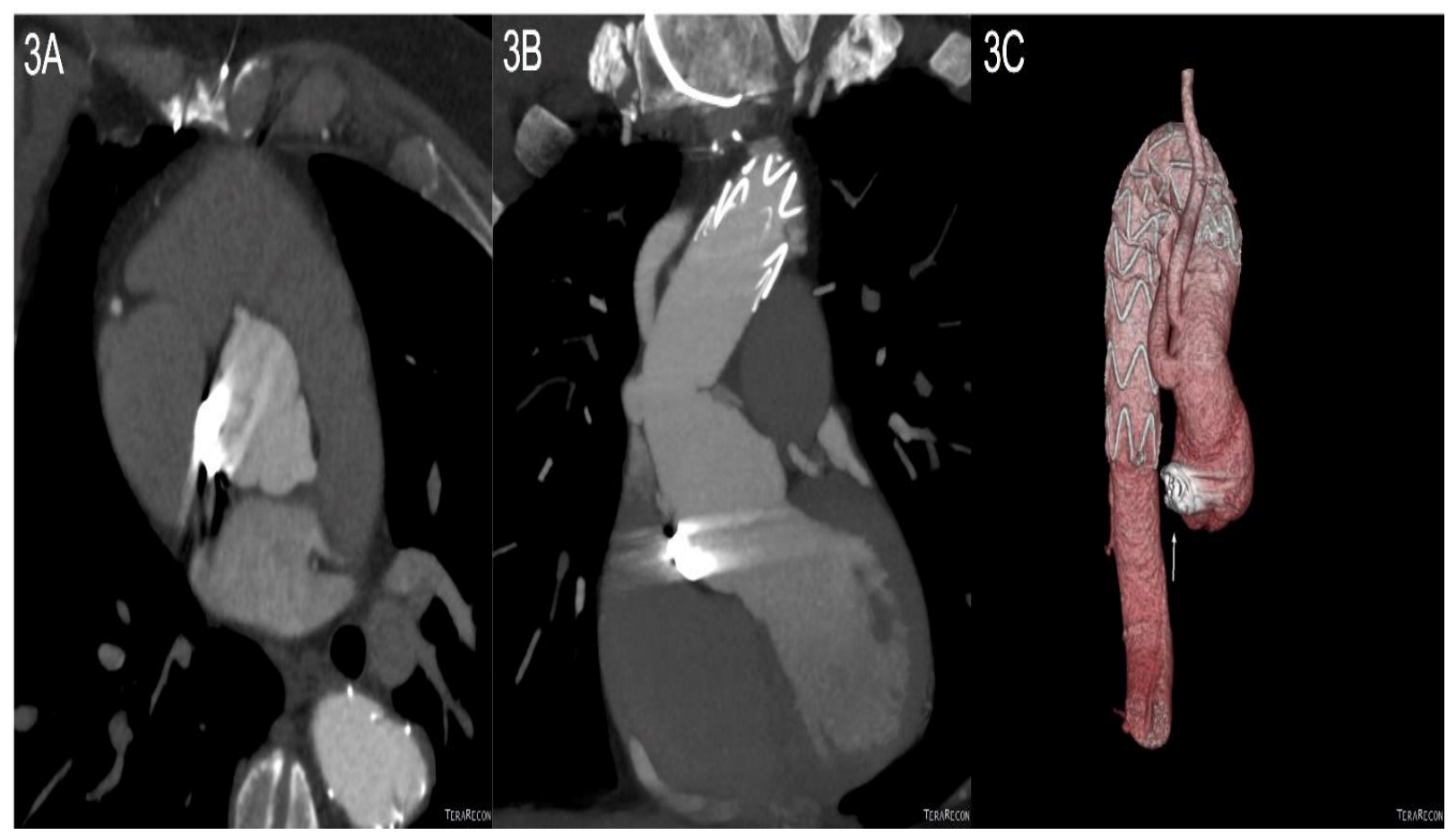

Figure 3. Three years CT follow-up reveal persistent exclusion of the pseudoaneurysm from blood flow in(3A) trasversal, (3B) coronal, and (3C) 3D reconstructions. 


\section{Discussion}

Reoperation on the thoracic aorta for aortic pseudoaneurysm was not without risk when it was attributed to hospital mortality of $6.9 \%^{1}$. Moreover, patient age and comorbidities can raise the risk of repeat surgery, and then a percutaneous treatment is often required.

The main concern about the presented post-surgical pseudoaneurysm was the proximity with coronary ostia that makes an interventional treatment more complex and rules out the possibility of deploying an endograft because the proximal landing zone does not have enough length and safety. Previously, several reports have shown the feasibility and safety of percutaneous embolization ofaneurysm and pseudoaneurysm in cerebrovascular and peripheral interventions [2-4].

Usually, plugs or coils are used for sac exclusion, but the first ones can be difficult to deploy, especially in tortuous anatomies. In contrast, the second ones are more manageable but can be time-consuming because of the need to detach numerous coils. Moreover, cases of successful aortic pseudoaneurysm occlusion with septal occluder devices were described [5-6].

The Penumbra Coil 400 (PC 400) is a large-volume platinum embolization device that, thanks to itslarger primary diameter, is designed to achieve higher packing density using fewer coils than conventional 10-system coils. The PC 400 system features an instant, single-click mechanical detachment system for ease of use.

PC 400 allowed us to deliver and reposition the coils as we want, maintaining microcatheter access while delivering large-volume coils. With few coils, we were able to ensure a complete embolization of the pseudoaneurysm quickly, safely, and with a good density pack due to the greater length, thickness, and softness. Three years CT follow-up highlights persistent exclusion of the pseudoaneurysm, demonstrating optimal acute and long-term procedural success.

\section{Conflict of interest}

The authors report no financial relationships or conflicts of interest regarding the content herein. The patient included in this manuscript has consented to the publication of this manuscript.

\section{References}

[1] Malvindi PG, van Putte BP, Heijmen RH, Schepens MA, Morshuis WJ. Reoperations for aortic false aneurysms after cardiac surgery. Ann Thorac Surg. 2010;90(5):1437-43.

[2] Popiela TJ, Brzegowy P, Łasocha B, Urbanik A. The effectiveness of Penumbra 400 micro-coils in the embolization of large cerebral aneurysms. Neurol Neurochir Pol. 2017;51(4):304-310.

[3] Marchiori E, Herten M, Bosiers M, Schwindt A, Bisdas T, Austermann M, et al. Effectiveness of Intra-Arterial Aneurysm Sac Embolization for Type Ia Endoleak after Endovascular Aneurysm Repair. J Vasc Interv Radiol. 2019 ;30(4):531-538.

[4] Wojtaszek M, Lamparski K, Wnuk E, Ostrowski T, Maciąg R, Rix T, et al. Selective occlusion of splenic artery aneurysms with the coil packing technique: the impact of packing density on aneurysm reperfusion correlated between contrastenhanced MR angiography and digital subtraction angiography. Radiol Med. 2019;124(6):450-459.

[5] Harky A, Khosravi A, Elam L, Colledge J, Kolvekar S. Percutaneous Intervention of a Rare Complication Post Cardiac Surgery. Ann Thorac Surg. 2017;104(1):e87.

[6] Lyen SM, Rodrigues JC, Manghat NE, Hamilton MC, Turner M. Endovascular closure of thoracic aortic pseudoaneurysms: A combined device occlusion and coil embolization technique in patients unsuitable for surgery or stenting. Catheter Cardiovasc Interv. 2016;88(7):1155-1169. 